\title{
Growth and Properties of ZnMnTe Nanowires
}

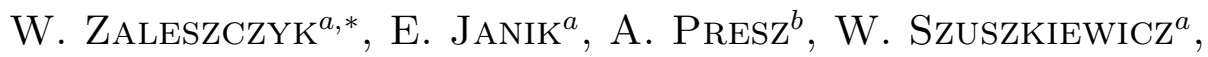
J.F. Morhange ${ }^{c}$, P. DŁużewski ${ }^{a}$, S. Kret ${ }^{a}$, H. Kirmse ${ }^{d}$, W. Neumann ${ }^{d}$, E. Dynowska ${ }^{a}$, J.Z. Domagata ${ }^{a}$,

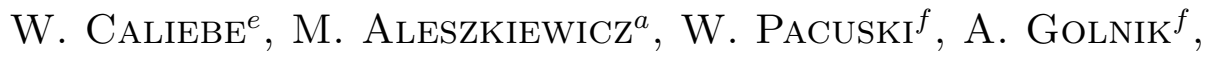
P. $\operatorname{Kossacki}^{f}$, L.T. BaczeWski ${ }^{a}$, A. PetroutchiK ${ }^{a}$, G. KARCZEWSKI ${ }^{a}$ AND T. WoJTOWICZ ${ }^{a}$

${ }^{a}$ Institute of Physics, Polish Academy of Sciences al. Lotników 32/46, 02-668 Warsaw, Poland

${ }^{b}$ Institute of High Pressure Physics, PAS

Sokołowska 29/37, 01-142 Warsaw, Poland

${ }^{c}$ Institut des Nanosciences de Paris, CNRS, Université Paris VI et Paris VII

140 rue de Lourmel, Paris 75015, France

${ }^{d}$ Humboldt-Universität zu Berlin, Institut für Physik, AG Kristallographie

Newtonstr. 15, 12489 Berlin, Germany

${ }^{e}$ Hasylab at DESY, Notkestr. 85, 22603 Hamburg, Germany

${ }^{f}$ Institute of Experimental Physics, Warsaw University

Hoża 69, 00-681 Warsaw, Poland

Catalytically enhanced growth of ZnMnTe diluted magnetic semiconductor nanowires by molecular beam epitaxy is reported. The growth is based on the vapor-liquid-solid mechanism and was performed on (001) and (011)-oriented GaAs substrates from elemental sources. X-ray diffractometry, scanning and transmission electron microscopy, atomic force microscopy, photoluminescence spectroscopy, and Raman scattering were performed to determine the structure of nanowires, their chemical composition, and morphology. These studies revealed that the obtained $\mathrm{ZnMnTe}$ nanowires possess zinc-blende structure, have an average diameter of about $30 \mathrm{~nm}$, typical length between 1 and $2 \mu \mathrm{m}$ and that $\mathrm{Mn}^{2+}$ ions were incorporated into substitutional sites of the ZnTe crystal lattice.

PACS numbers: 61.46.Df, 68.65.La, 78.55.Et, 78.67.Bf, 81.15.Hi

*corresponding author; e-mail: wzal@ifpan.edu.pl 


\section{Introduction}

The studies of semiconductor nanowires (NWs) have been recently intensified due to the hope of using these 1D structures as "building blocks" for nanoscale electronic and photonic devices. A broad range of semiconductor nanowires, also including binary II-VI group materials, have been grown using chemical vapor deposition, thermal evaporation, solvothermal synthesis, and molecular beam epitaxy (MBE) based on the gold-catalyzed vapor-liquid-solid (VLS) mechanism [1]. However, a synthesis of only a few ternary compound semiconductor NWs, such as ZnCdSe [2], CdMnS [3-7], and ZnMnS [7-10] has been reported so far.

In the present work we report on the first successful growth and properties of ZnMnTe NWs. These ZnMnTe NWs are potentially attractive for spintronic nanodevice applications because the heavily $p$-type doped ZnTe crystals alloyed with MnTe exhibit at low temperature the paramagnetic-to-ferromagnetic phase transition [11].

\section{Experiments}

ZnMnTe NWs were grown in an EPI 620 MBE system equipped with low temperature effusion cells and reflection high energy electron diffraction (RHEED). The growth process based on the Au-catalyzed vapor-liquid-solid mechanism was performed simultaneously on (001) and (011)-oriented GaAs substrates. The optimum growth temperature of the ZnMnTe NWs was found to be in the range of $420-500^{\circ} \mathrm{C}$. NWs with an average diameter of about $30 \mathrm{~nm}$ and a typical length from 1 to $2 \mu \mathrm{m}$ were grown by this method.

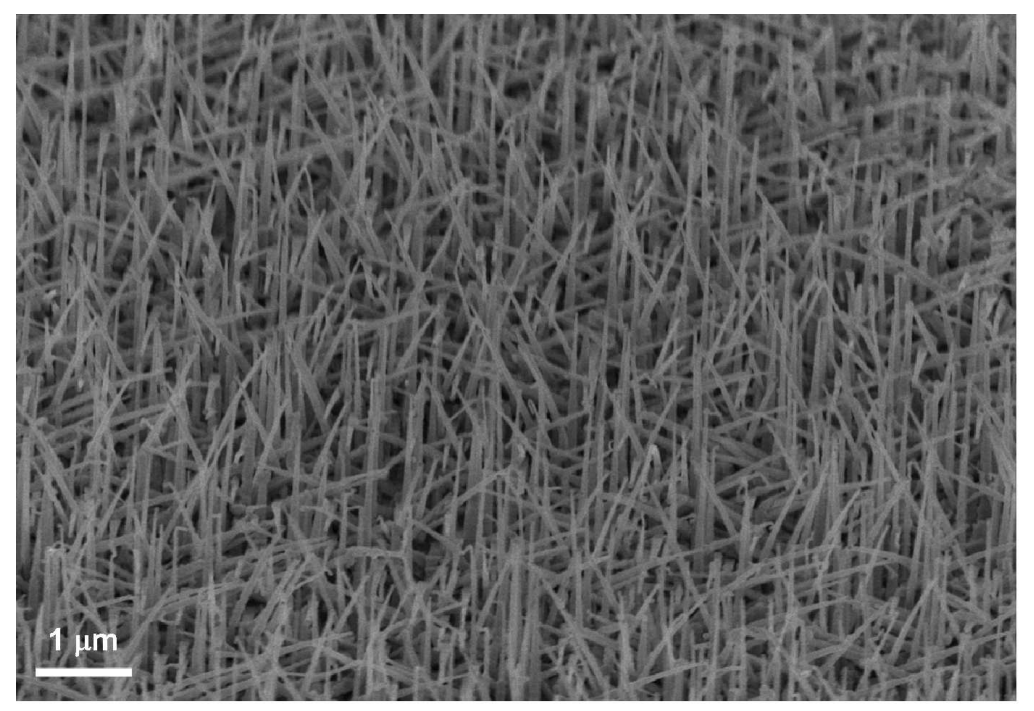

Fig. 1. FE-SEM image of ZnMnTe nanowires grown on a (100)-oriented GaAs substrate viewed at an angle of $45^{\circ}$ to the substrate normal direction. 
After the growth several images have been taken with the use of a field-emission scanning electron microscope (FE-SEM Leo 540). FE-SEM image of typical ZnMnTe NWs grown on (001)-oriented GaAs substrate is shown in Fig. 1. The structural characterization of NWs was performed by means of X-ray diffraction (XRD), using the characteristic radiation of Fe: $K_{\alpha}(\lambda=1.9373 \AA)$ and $K_{\beta}$ $(1.75661 \AA)$. The samples were examined also by atomic force microscopy (AFM - Nanoscope III MultiProbe (DI)) and high resolution transmission electron microscopy (HRTEM — JOEL JEM 2000EX). The studies of optical properties of ZnMnTe NWs were done by the Raman scattering and photoluminescence (PL) measurements. All Raman scattering measurements were performed in a quasibackscattering geometry using a Jobin-Yvon U1000 spectrometer equipped with holographic gratings, a S20 photomultiplier, and a photon counting system.

\section{Results and discussion}

The XRD measurements allowed us to determine the chemical composition of studied ZnMnTe NWs based on the change of lattice parameter. This determination is quite accurate since in the case of NWs the influence of substrate induced strain on lattice parameter of ZnMnTe is insignificant. This is because in the NW geometry the efficient strain relaxation due to the proximity to the open side surface takes place, as was previously observed for $\mathrm{InP} / \mathrm{InAs}$ system [12]. Concentrations of $\mathrm{Mn}$ in the range from 12 to $30 \%$ were found for samples grown with different Mn fluxes. Analysis of diffraction patterns also shows that crystallographic orientation of the substrate imposes the orientation of the nanowires. In Fig. 2 an example of diffraction pattern obtained for the sample of the highest Mn concentration is presented. One can see there the strong 002 and 004 reflections

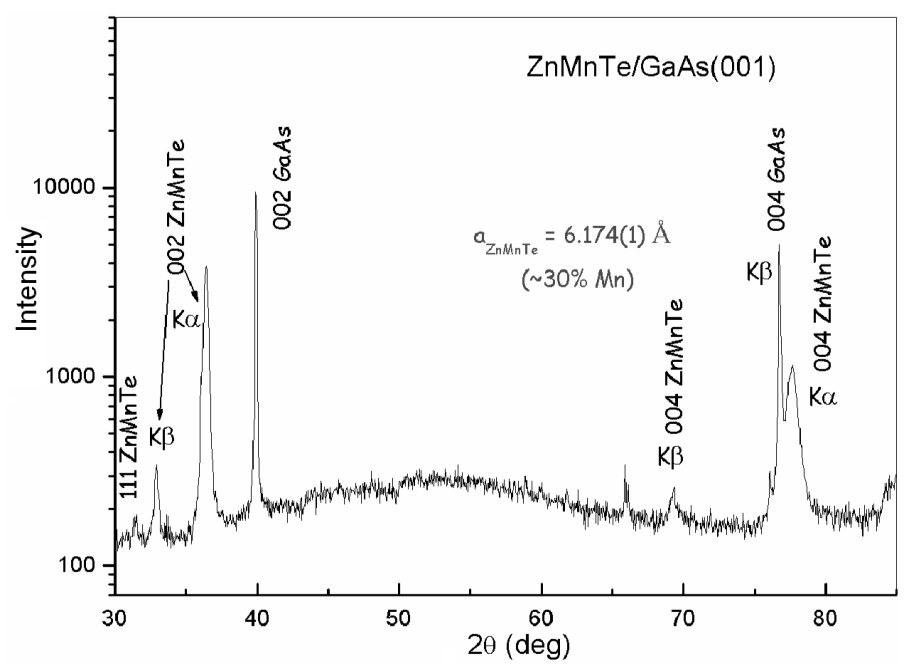

Fig. 2. Typical XRD pattern of as-grown ZnMnTe nanowires on GaAs (100) substrate. The Mn concentration for this sample was determined to be around $30 \%$. 
from ZnMnTe NWs that were obtained by adjusting sample orientation so as to maximize analogous reflections from the GaAs substrate. This indicates that the (001) lattice planes in nanowires are parallel to the (001) lattice planes of the substrate.

Figure 3 presents typical Raman spectra collected from ZnTe and ZnMnTe NWs samples within the frequency range from 190 to $225 \mathrm{~cm}^{-1}$ and containing features related to the ZnTe longitudinal optical (LO)-phonon $\left(205 \mathrm{~cm}^{-1}\right)$ and the ZnMnTe LO-phonon $\left(212 \mathrm{~cm}^{-1}\right)$, respectively. The frequencies of LO-phonons and the width of the Raman lines correspond well to the values typical of high-quality ZnTe and ZnMnTe bulk single crystals or epilayers. These two spectra (Fig. 3) are optical proof of the high structural quality of the NWs and confirmation of the fact that $\mathrm{Mn}^{2+}$ ions are situated at a tetrahedral lattice site, and hence that mixed crystal diluted magnetic semiconductors (DMS) nanowires were formed.

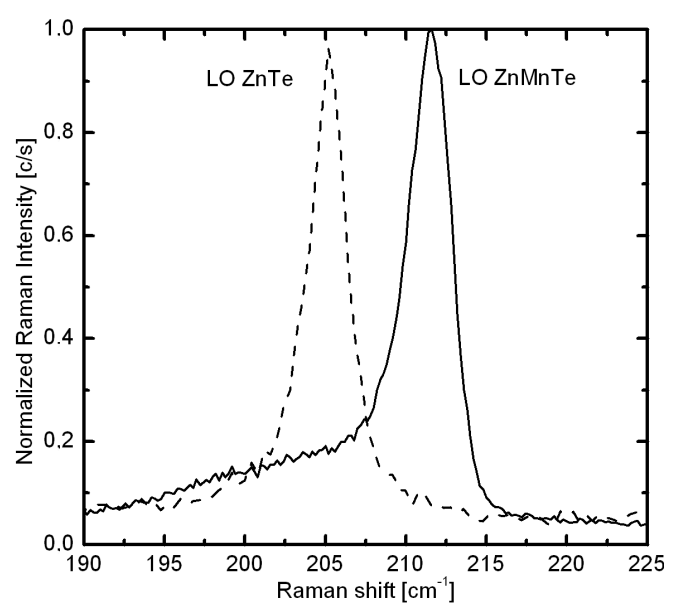

Fig. 3. Raman scattering spectra of ZnTe NWs deposited onto Si wafer (dashed line) and of as-grown ZnMnTe/GaAs(100) NWs (solid line) samples. Data were taken at a temperature of $15 \mathrm{~K}$ with the use of the $\mathrm{Ar}^{+}$laser excitation line $\lambda=514.5 \mathrm{~nm}$ and with the laser power of $50 \mathrm{~mW}$.

PL spectra of either as-grown NWs on GaAs substrate or of NWs extracted from the substrate and deposited onto Si wafer were measured. The AFM image of ZnMnTe NWs resting on Si wafer is presented in Fig. 4a. TEM investigations (Fig. 4b) confirm the structural perfection of ZnMnTe NWs. In particular, we do not observe the stacking faults characteristic of ZnTe NWs [1]. However, most of the NWs were bent, which suggests the presence of the surface or internal stress. The PL spectra of such samples show a peak centered at an energy of about $2 \mathrm{eV}$ which is characteristic of intra-atomic transition of $\mathrm{Mn}^{2+}$ ion located at a substitutional Zn site of ZnTe crystal. The photoluminescence studies of ZnMnTe NWs will be discussed in detail in a separate paper. 


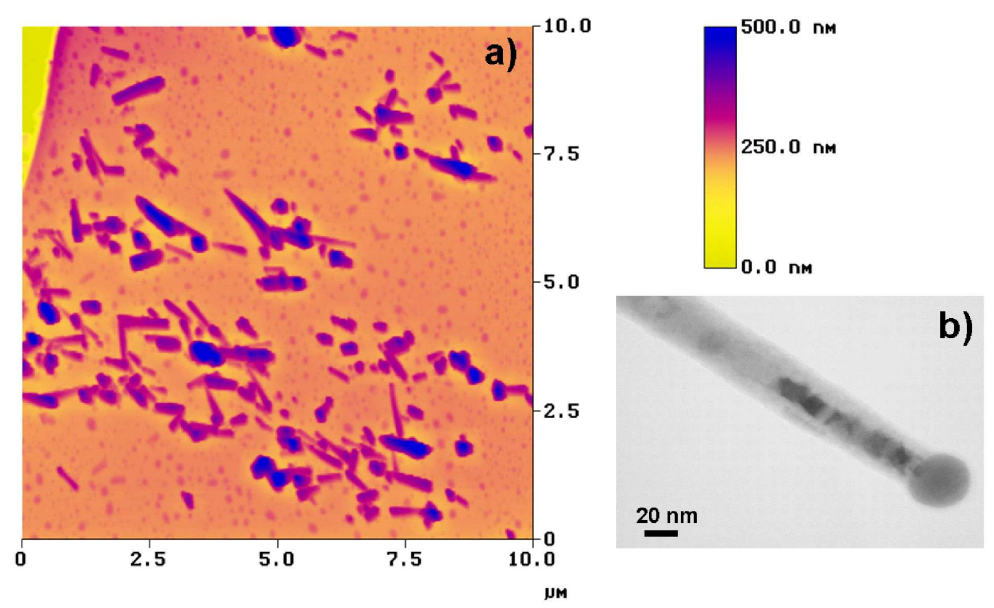

Fig. 4. (a) AFM image of ZnMnTe NWs removed from GaAs substrate and deposited onto Si wafer, (b) TEM image of the top part of single ZnMnTe NW with the Au particle visible at the tip.

\section{Conclusions}

In summary, we have reported MBE growth of high quality $\mathrm{ZnMnTe}$ nanowires with manganese concentration between 12 and 30\%. Investigations of these NWs indicate that $\mathrm{Mn}^{2+}$ atoms are introduced into substitutional sites of the ZnTe matrix. The growth of nanowires containing a large concentration of $\mathrm{Mn}$ from a semiconductor that can be also heavily $p$-type doped opens new perspectives for applications in nanospintronics.

\section{Acknowledgments}

This research was partially supported by the Ministry of Science and Higher Education (Poland) through grant No. N507 030 31/0735 and by the Network "New materials and sensors for optoelectronics, information technology, energetic applications and medicine".

\section{References}

[1] E. Janik, J. Sadowski, P. Dłużewski, S. Kret, L.T. Baczewski, A. Petroutchik, E. Łusakowska, J. Wróbel, W. Zaleszczyk, G. Karczewski, T. Wojtowicz, A. Presz, Appl. Phys. Lett. 89, 133114 (2006).

[2] A. Colli, S. Hofmann, A.C. Ferrari, F. Martelli, S. Rubini, C. Ducati, A. Franciosi, J. Robertson, Nanotechnology 16, S139 (2005).

[3] Ch. Cheng, G. Xu, H. Zhang, H. Wang, J. Cao, H. Ji, Mater. Chem. Phys. 97, 448 (2006).

[4] S.K. Dae, Y.L. Jin, W.N. Chan, W.Y. Sang, Y.K. Shin, P. Jeunghee, J. Phys. Chem. B 110, 18262 (2006). 
[5] J.Z. Liu, P.X. Yan, G.H. Yue, J.B. Chang, D.M. Qu, R.F. Zhuo, J. Phys. D, Appl. Phys. 39, 2352 (2006).

[6] A.V. Kouzema, M. Froba, L. Chen, P.J. Klar, W. Heimbrodt, Adv. Funct. Mater. 15, 168 (2005).

[7] P.V. Radovanovic, C.J. Barrelet, S. Gradecak, Q. Fang, C.M. Lieber, Nano Lett. 5, 1407 (2005).

[8] Y.Q. Chang, D.P. Yu, H.Z. Zhang, Z. Wang, Y. Long, W.J. Qiang, Nanotechnology 17, 1999 (2006).

[9] H.J. Yuan, X.Q. Yan, Z.X. Zhang, D.F. Liu, Z.P. Zhou, L. Cao, J.X. Wang, Y. Gao, L. Song, L.F. Liu, X.W. Zhao, X.Y. Dou, W.Y. Zhou, S.S. Xie, J. Cryst. Growth 271, 403 (2004).

[10] P.G. Jian, W. Jin, X.Z. Hao, W. Xun, P. Qing, D.L. Ya, Adv. Funct. Mater. 15, 303 (2005).

[11] D. Ferrand, J. Cibert, A. Wasiela, C. Bourgognon, S. Tatarenko, G. Fishman, T. Andrearczyk, J. Jaroszyński, S. Koleśnik, T. Dietl, B. Barbara, D. Dufeu, Phys. Rev. B 63, 085201 (2001).

[12] M.T. Björk, B.J. Ohlsson, T. Sass, Q. Fang, A.I. Persson, C. Thelander, M.H. Magnusson, K. Deppert, L.R. Wallenberg, L. Samuelson, Nano Lett. 2, 87 (2002). 